\title{
Uso de la Tecnología de la Información y Comunicación en educación virtual y su correlación con la Inteligencia Emocional de docentes en el Ecuador en contexto COVID-19
}

\author{
Zambrano Vacacela Luis Leonardo ${ }^{1}$ \\ leozamv@hotmail.com \\ ${ }^{1}$ Universidad Nacional de Educación (UNAE), 030104, Azogues, Ecuador.
}

DOI: $10.17013 /$ risti.40.31-44

\begin{abstract}
Resumen: El presente estudio propone una investigación mixta de tipo no experimental, transeccional, correlacional con alcance descriptivo, para evidenciar el nivel de conocimiento y uso de las Tecnologías de Información y Comunicación (TIC) en educación virtual y su correlación con la Inteligencia Emocional (I.E) de 485 profesores de nivel inicial, primario y secundario; además, pretende revelar el contexto en el que se está desarrollando el proceso de enseñanza-aprendizaje en relación con la emergencia sanitaria COVID-19. De los resultados se destaca que existen pocas habilidades en el uso de las TIC, a lo que se añade que la mayoría de docentes carecen de infraestructura tecnológica y acceso a internet; también, que las profesoras tienen menos fortalezas emocionales, y que existe muy baja correlación entre el manejo de las TIC y la I.E. Finalmente, se destaca que la aplicación WhatsApp ha sido transformada de medio de comunicación instantánea a plataforma de aprendizaje.
\end{abstract}

Palabras-clave: TIC; educación virtual; inteligencia emocional; enseñanza aprendizaje.

Use of Information and Communication Technology in virtual education and its correlation with the Emotional Intelligence of Teachers in Ecuador in a COVID -19 context

Abstract: The present study proposes a mixed investigation of a non- experimental
transactional correlational type with a descriptive scope in order to demonstrate
the level of knowledge and use of Information and Communication Technology
(ICT) in virtual education and its correlation with Emotional Intelligence (EI) of
485 teachers of initial, primary and secondary level. In addition, it aims to reveal
the context in which the teaching-learning process is being developed in relation
to the COVID-19 emergency. The results show that there are few ICT skills being
utilized. Additionally, most teachers lack technological infrastructure and internet
access. This study also indicates that female teachers have less emotional strengths
and there is a very low correlation between the use of ICT and EI. Finally, it is 
highlighted that the WhatsApp application has been transformed from a means of instant communication to a learning platform.

Keywords: ICT; virtual education; emotional intelligence; teaching-learning.

\section{Introducción}

La emergencia sanitara originada por el corona virus (COVID-19), causante del síndrome respiratorio agudo grave (SARS-COV-2) que afecta a varios países a nivel mundial (Organización Mundial de la Salud, 2020), ha llevado a pensar y accionar de formas distintas los ambientes en donde el ser humano se vincula con la sociedad: salud, educación, economía y todos los demás contextos; como consecuencia, las personas han obtenido una perspectiva diferente de la vida, ya sea en el ámbito social, laboral o familiar, lo cual influye directamente en la forma de trabajar y en las emociones.

En este sentido y dada la cuantía de los casos positivos detectados respecto al número de habitantes, Ecuador es el segundo país más afectado por el virus COVID-19 en América Latina (Hallo et al., 2020). Esto ha obligado al Estado a adoptar medidas para salvaguardar la salud de los ciudadanos: confinamiento, uso de la vestimenta adecuada y aislamiento social, entre otras (Inca \& Inca, 2020).

En lo que respecta el ámbito educativo, se destaca en ese país el importante papel que han adquirido los entornos virtuales para dar continuidad a la educación, los cuales hasta ahora solo habían sido considerados relevantes en la educación superior (Suasnabas et al., 2018); no tanto en los niveles de inicial, primaria y secundaria, en los que eran utilizados de manera esporádica.

A pesar de la extensa variedad de formas de enseñar y aprender que emergen de las nuevas tecnologías, en el Ecuador no se ha podido verificar la existencia de un plan de contingencia a nivel gubernamental que, mediante la modalidad virtual, supla las necesidades educativas orientadas a fomentar las capacidades individuales, emocionales y motivacionales del alumnado, así como del resto de actores que intervienen en el proceso de enseñanza-aprendizaje; el fomento de estas capacidades ha sido una demanda constante por parte de los estudiantes, los profesores y la sociedad en general (Renés, 2018).

La escasa planificación sobre esta materia se evidencia en la precariedad que, en lo referente a los entornos virtuales, muestran las instituciones educativas en los tres primeros niveles. Tanto las autoridades como los profesores, estudiantes, padres de familia y colectividad, no han sido objeto de la suficiente capacitación-cualificación; tampoco disponen de los recursos tecnológicos-infraestructura, ni de los psicológicosemocionales requeridos para adoptar, de manera estricta, las medidas recomendadas para enfrentar este acontecimiento mundial (Rodriguez-Morales et al., 2020).

Desde esta perspectiva, es preciso medir el nivel emocional del cuerpo docente para establecer su correlación con el manejo de las Tecnologías de Información y Comunicación (TIC) en la modalidad de educación no presencial; de esta forma, se pretende percibir cómo se sienten emocionalmente frente a las limitaciones que presenta la educación y la sociedad en el manejo de entornos virtuales. 


\section{Uso de las TIC en la educación virtual}

Para garantizar la calidad de la educación virtual es necesario que todo el sistema educativo se adapte a ese nuevo espacio, lo cual implicaría el diseño de nuevas estructuras, métodos e instrumentos. Para ello, con fundamento en las políticas educativas, se deben crear los escenarios propicios para que docentes, estudiantes, padres de familia, autoridades y colectividad, tengan acceso a la necesaria capacitación y cualificación en el uso y manejo de las Tecnologías de Información y Comunicación (Rodríguez, 2016).

Existen diversos modelos para evaluar la calidad (Marciniak \& Gairín, 2018), así como múltiples estrategias pedagógicas y compendios de buenas prácticas docentes que podrán ser utilizados dependiendo del contexto (Bolívar \& Dávila, 2016), pero que en todo caso deberán estar orientados al cumplimiento de los objetivos de aprendizaje, así como a proveer las competencias que debe alcanzar el estudiantado que aprende bajo la modalidad virtual.

\subsection{Formación del profesorado}

Es limitada la capacitación de los docentes en cuanto a los aspectos pedagógicos asociados con la utilización de los medios virtuales para fines educativos. La falta de inserción de las Tecnologías de Información y Comunicación (TIC) en el proceso de enseñanzaaprendizaje, resulta de la carencia de espacios que fomenten el uso de las TIC, lo cual está dificultando la adquisición de habilidades y destrezas pedagógicas relacionadas con la didáctica y el acompañamiento en entornos virtuales. Como consecuencia, solamente una pequeña parte de la población de docentes, impulsados por la iniciativa personal y la creatividad, han encontrado la forma de insertar herramientas de la web 2.0 y las redes sociales al proceso (Balladares-Burgos, 2018).

La educación debe ejecutar acciones que propicien una alfabetización digital, ya que a partir de allí se pueden generar otros ambientes en los que el estudiante se motive para aprender, utilizando recursos interactivos, visuales y sonoros, entre otros. Por esta razón y para dar respuesta a las necesidades educativas del contexto, es preciso que los maestros se capaciten en el correcto uso de las TIC (Gómez \& Trigueros, 2017).

\subsection{Planificación didáctica en entornos virtuales}

Para desarrollar de forma eficaz el proceso de enseñanza-aprendizaje en entornos virtuales, es importante considerar todas las variables que intervienen; entre estas se destacan: los roles del maestro y el estudiante como constructores del aprendizaje; las estrategias y técnicas a implementarse, y los recursos que se requieren para alcanzar resultados óptimos en el proceso educativo (Manrique-Losada et al., 2020). Estos factores deben tener relación directa con las necesidades, objetivos, destrezas y con la realidad del contexto en donde se desarrolla la educación.

Sobre este particular, la planificación debe responder a los principios de: (a) perceptibilidad, para que los estudiantes puedan apreciar toda la información disponible; (b) operatividad, para facilitar la navegación; (c) comprensibilidad, para entender los contenidos que demanda el proceso educativo; y (d) robustez, para proporcionar 
una amplia gama de opciones para acceder a la información disponible, desde la ayuda técnica hasta la forma de utilizar los múltiples recursos disponibles (Mogollón et al., 2017).

En lo que respecta a la planificación didáctica, se evidencia el escaso conocimiento de los profesores para poder desarrollarla en Entornos Virtuales de Aprendizaje (EVA), lo cual refleja la necesidad de formar a los maestros en esta temática; no obstante, a pesar de la apertura que demuestran para cualificarse, se observan limitaciones administrativas de las instituciones para acceder a redes e impulsar la actualización docente en EVA (Granados et al., 2017).

\subsection{Desarrollo de la planificación didáctica}

Durante el desarrollo de la educación virtual se enfatiza la necesidad de generar una eficiente comunicación entre el docente y el estudiante, en donde el maestro, por medio de la retroalimentación (feedback) individual o grupal, clara y precisa, pueda motivar al aprendizaje, facilitando que el educando responda con una participación efectiva. Expresado en otras palabras, la comunicación es un elemento indispensable para el trabajo en plataformas virtuales educativas (Cabanillas et al., 2020); solo así se logrará alcanzar las competencias que demanda el currículo, teniendo a disposición diversos recursos como: foros, sección de anuncios, mensajería, entre otros (Alemany \& Martínez, 2017a).

En este sentido, el docente debe plantear una planificación sistemática fundamentada en los contenidos a impartir, en los conocimientos que debe promover, en la formulación de instrucciones claras para lograr el entendimiento, y en el desarrollo de las estrategias para fomentar la interacción en el proceso, siendo a partir de allí que se podrá estructurar el aula virtual (López et al., 2019) mediante el uso correcto de las TIC (ZambranoVacacela y Yautibug-Chimbolema, 2020).

\subsection{Evaluación de los aprendizajes en educación virtual}

Para satisfacer los requerimientos de la evaluación virtual existe en la web una variedad de recursos que ayudan a verificar el aprendizaje interiorizado por los estudiantes; entre ellos destacan: cuestionarios en línea, juegos interactivos, puzzles, vídeos, entre otros (Alemany \& Martínez, 2017b), de tal forma que el docente puede optar por el que considere que mejor se adapta a la realidad de su contexto educativo y, con base en los resultados, reorientar, fomentar o fortalecer la forma en la que está impartiendo su cátedra.

En ese marco, el profesor debe contar con una formación integral, profesional, que le permita enfrentarse y dar respuesta a las necesidades que demanda el proceso de enseñanza-aprendizaje en su contexto educativo; siempre en sintonía con el manejo de las herramientas que ofrecen las Tecnologías de Información y Comunicación (TIC) para la virtualización de evaluaciones que serán desarrolladas por el estudiantado (Mesa et al., 2018). 


\subsection{Inteligencia emocional}

Los aspectos que influyen directamente sobre el bienestar constituyen la base de la percepción de las cosas y de la forma de pensar que poseen los seres humanos (García, 2016). Estos puntos de vista se encuentran estrechamente relacionados con la inteligencia emocional. Tener fortaleza sobre esta, conduce a comprender que alcanzar el éxito no es sinónimo de adquirir mayores bienes materiales, sino de alcanzar la felicidad mediante la manipulación efectiva de las emociones para estar bien consigo mismo, con los demás y con los acontecimientos.

Las investigaciones desarrolladas sobre inteligencia emocional demuestran que está relacionada directamente con la eficiencia en el mundo laboral. Tanto de manera individual como colectiva, representa un factor determinante en el empoderamiento del trabajador para la labor que desempeña; razón importante por la que se debería considerar el fortalecimiento de este constructo permanentemente (Goleman, 2010). Igualmente, existe un vínculo estrecho entre el liderazgo, la inteligencia emocional, el ambiente y el rendimiento de una persona, incidiendo también sobre los estilos de liderazgo de los individuos que adoptan este papel (Goleman, 2018).

Desde esta perspectiva, Merchán \& González (2012) afirman que el docente debe ser inteligente emocionalmente, pues esto favorece las relaciones entre la comunidad educativa y previene el agotamiento causado por el estrés crónico que sufren muchos maestros; este hecho justifica la necesidad de que sea potenciada en todo el sistema de educación, incluso desde la formación del magisterio. No obstante, a pesar de los estudios que de forma recurrente demuestran la importancia de la I.E. en el desarrollo humano, aún no existen programas gubernamentales que impulsen el fortalecimiento de este constructo en todos los actores del sistema educativo ecuatoriano.

Manteniendo este hilo conductor, varios autores clasifican la inteligencia emocional en: atención, claridad y reparación (Salovey et al., 1995); autoconocimiento emocional, autocontrol emocional, automotivación, empatía, relaciones inter-personales, intrapersonales, manejo del estrés y adaptabilidad (Bar-On, R. \& Parker, J., 200o; Goleman, 2007; Ugarriza \& Pajares-Del-Águila, 2005). De lo anterior se destaca que aun cuando existen diferentes clasificaciones, todas están direccionadas hacia las acciones que ejecutan las personas sobre sí mismas y sobre las demás, con base en el manejo eficiente de las emociones.

\section{Propósito de la investigación}

El propósito de la investigación es presentar un análisis descriptivo del contexto en el que se está desarrollando la educación básica y secundaria en el Ecuador, en relación con el conocimiento y manejo de las Tecnologías de Información y Comunicación (TIC) en la educación virtual, y su correspondencia con la Inteligencia Emocional. A partir de allí se pretende evidenciar las limitaciones que poseen los maestros para ejecutar la labor docente ante la emergencia sanitaria COVID-19, y conocer la manera como este fenómeno influye en las emociones. 


\subsection{Objetivos}

- Identificar el nivel de conocimiento y uso de las Tecnologías de Información y Comunicación (TIC) que aplican los docentes en la educación virtual.

- Identificar el nivel de inteligencia emocional de los profesores en relación al manejo de las TIC en educación virtual.

- Demostrar la correlación entre las habilidades para el manejo de la TIC en educación virtual y la inteligencia emocional de los profesores.

\section{Diseño y método}

El estudio parte de un diseño de investigación mixto, de tipo no experimental, transeccional, con alcance descriptivo y correlacional (Hernández et al., 2014). La data fue obtenida mediante la aplicación de un cuestionario unificado para medir la inteligencia emocional y las habilidades de los docentes en cuanto al uso de las TIC en el contexto de la educación virtual. Dicho instrumento se aplicó por una sola vez entre los meses de abril a junio de 2020.

\subsection{Muestra}

Tal como se muestra en la Tabla 1, en esta investigación participaron 485 profesores con edades comprendidas entre los 20 y los 60 años, de los cuales el $41 \%$ son de sexo masculino y el 59\% femenino. Laboran en los tres niveles de educación: inicial 4,9\%; básica $81,4 \%$ y bachillerato $13,2 \%$; en instituciones unidocentes (un solo profesor para todos los grados al mismo tiempo) 7\%; pluridocentes (un docente para dos o tres grados al mismo tiempo) 31,1\% y completa (un educador para cada grado) 61,9\%. El 86\% de las instituciones en donde laboran los maestros son de sostenimiento fiscal; fiscomisional el $12 \%$, y particular el $2 \%$. Los establecimientos que pertenecen al sector urbano representan el $41,9 \%$ y al sector rural el 58,1\%. De la totalidad de la población, el 71,3\% imparte 'Educación Intercultural' y el 28,7\% 'Educación Intercultural Bilingüe'. Finalmente, se destaca que en la ejecución del cuestionario unificado colaboraron maestros y maestras de 20 provincias de las 24 que existen en el Ecuador.

\begin{tabular}{llllllllllll}
\hline Sexo & F & Edad & F & Nivel & F & Inst. & F & Sost. & F & Lug. & F \\
\hline$M$ & 199 & $20-25$ & 34 & Inic. & 24 & Unidoc. & 34 & Fiscal & 419 & Urb. & 203 \\
\hline$F$ & 286 & $26-55$ & 446 & Bas. & 395 & Pluridoc. & 151 & Fiscom. & 58 & Rur. & 282 \\
\hline & - & $56-60$ & 5 & Sec. & 66 & Compl. & 300 & Priv. & 8 & & - \\
\hline Total & 485 & & & & & & & & & & \\
\hline
\end{tabular}

Tabla 1 - Descripción de la población

\subsection{Instrumentos}

Para la recolección de datos se utilizaron dos instrumentos unificados en un solo cuestionario aplicado en línea: 
El primero fue el Trait Meta Mood Scale-24 (TMMS; Salovey \& Mayer, 1989-90). Adaptado al castellano por (Fernández-Berrocal et al., 1998), posee una escala de Likert que mide tres variables en 24 preguntas: la primera variable es 'atención' para evidenciar aspectos relacionados a la intrapersonalidad; la segunda es 'claridad' que mide aspectos referentes a las emociones como el estado de ánimo y el estrés; y la tercera es 'reparación', que muestra cuestiones relacionadas con la adaptabilidad y el manejo efectivo de las emociones. Este instrumento ha sido validado en América Latina dando resultados adecuados para su aplicación; posee una confiabilidad del 95\% y un coeficiente Alfa de Cronbach mayor al 85\%, lo que evidencia buena consistencia y homogeneidad en todas sus dimensiones (Espinoza-Venegas et al., 2015).

El segundo instrumento es un cuestionario de elaboración propia, orientado a recoger datos relacionados con la educación virtual y el uso de las TIC en el proceso de enseñanzaaprendizaje. Este cuestionario se divide en cuatro variables: acceso a internet, manejo de plataformas virtuales, planificación de clases virtuales y evaluación de aprendizajes mediante el uso de la plataforma virtual; para lo que se formularon 15 ítems con opciones de respuesta múltiple, tal como se indica a continuación:

Acceso a internet:

1. Para impartir clases virtuales, tengo internet (permanentemente; esporádicamente; nunca).

2. Del total de su clase, indique según su criterio el porcentaje de estudiantes que tienen acceso a internet: (todos [76-100\%]; 3/4 partes [51-75\%]; mitad [2650\%]; 1/4 parte [1-25\%]; ninguno [o\%]).

3. Los estudiantes cumplen en el tiempo establecido las actividades enviadas a través de la plataforma: (todos [76-100\%], 3/4 partes [51-75\%], mitad [26-50\%], $1 / 4$ parte $[1-25 \%]$, ninguno [o\%]).

Manejo de plataformas virtuales:

4. La institución en la que labora tiene alguna plataforma específica para generar el proceso enseñanza aprendizaje, seleccione una opción: (Blackboard, Moodle, Idukay, Microsoft Teams, no tiene plataforma virtual, otra).

5. Seleccione el sistema de gestión del aprendizaje que utiliza como herramienta para impartir sus clases (Blackboard; Microsoft Teams; Zoom; WhatsApp; otra).

6. Según su criterio, ¿cuál es el nivel de manejo de entornos virtuales por parte de sus estudiantes? (alto, medio, bajo, nulo);

7. Según su criterio, ¿̇cuál es el nivel de manejo de entornos virtuales por parte de los padres de familia de sus estudiantes? (alto; medio; bajo; nulo).

8. Según su criterio, ¿cuál es el nivel de manejo de entornos virtuales que usted posee como docente? (alto; medio; bajo; nulo).

9. Usted como docente ¿ha recibido capacitación para el manejo de entornos virtuales en educación? ( $s i$; no).

10. ¿Qué institución le ha brindado capacitación relacionada con el manejo de entornos virtuales en educación? (Ministerio de Educación; universidad a la que asisto o asistí: aprendí solo en casa; realicé cursos en otras instituciones; no manejo plataformas virtuales en educación; otra). 
Planificación de clases virtuales:

11. Cuando imparte clases virtuales expone los objetivos y competencias a adquirir (si; no; a veces).

12. Las actividades que deben resolver sus estudiantes ¿pueden ser desarrolladas sin la necesidad de tener conexión permanente a internet? (si; no; a veces).

13. Como recursos para el desarrollo de aprendizajes individuales, Usted como docente utiliza: (contenidos interactivos en plataforma; resolución de actividades sin conexión a internet; investigación de contenidos en internet; foros en plataforma; clase magistral en plataforma; otro).

Evaluación de los aprendizajes:

14. Para evaluar los aprendizajes alcanzados, Usted como docente utiliza (cuestionarios en línea; trabajos o tareas; otra).

15. Si pudiera escoger la modalidad de enseñanza aprendizaje ¿̇cuál seleccionaría? (presencial; semi presencial; virtual; otra).

\subsection{Trabajo de campo}

Para la recolección de datos, los dos instrumentos ya detallados se unificaron en un solo cuestionario en la herramienta Google Forms online; el cual fue enviado por correo electrónico a varios docentes a nivel nacional (24 provincias en el Ecuador) a quienes se les solicitó que fueran respondidos de forma completa.

\section{Análisis de datos}

Los datos fueron analizados con el programa estadístico SPSS versión 22, mediante el cual se hizo el análisis de frecuencias para la descripción del nivel de conocimiento y uso de las TIC en entornos educativos virtuales, y establecer su correspondencia con la Inteligencia Emocional. Para verificar la correlación entre ambas variables se utilizó el coeficiente de correlación de Spearman.

\subsection{TIC en educación virtual}

Sobre las habilidades en el uso de las Tecnologías de Información y Comunicación se destaca que un alto porcentaje de docentes $(74,4 \%)$ tiene un nivel medio; sin embargo, un $6,4 \%$ aducen que no tienen conocimiento sobre las TIC para entornos virtuales y el 19,2\%, que también es un número bajo, tienen un nivel alto. (Tabla 2).

\begin{tabular}{ll}
\hline Nivel & F \\
\hline Bajo & 31 \\
\hline Medio & 361 \\
\hline Alto & 93 \\
\hline Total & 485 \\
\hline
\end{tabular}

Tabla 2 - Nivel de manejo de TIC en educación virtual 
En relación a estos resultados, para el aprendizaje sobre el uso de las TIC en entornos virtuales, el 31,3\% de los profesores manifestó que desarrollaron un autoaprendizaje en la web; el 11,1\% solicitó ayuda a sus familiares; el 19,4\% aprendió en la universidad y el $37,4 \%$, lo hizo mediante cursos propuestos por el Ministerio de Educación o la institución en donde labora.

Frente al nivel medio de las habilidades en el manejo de la TIC para entornos virtuales, se evidencia que el $93,2 \%$ de los maestros no disponen de una plataforma virtual para impartir sus clases; solamente el 6,8\% puede manejar y acceder esporádicamente a Blackboard (o,8\%), Idukay (o,6\%), Moodle (5,2\%) y Zoom (0,2\%).

En respuesta a esta carencia, el 71,1\% de los encuestados utilizan WhatsApp como herramienta para ejecutar el proceso de enseñanza-aprendizaje; esto debido a que es una aplicación gratuita, de fácil acceso, mediante la que se puede interactuar para tratar de cumplir con dicho proceso. Este resultado no coincide con los datos obtenidos sobre el manejo de las TIC en entornos virtuales, pues en la web existe una variedad de recursos que los docentes podrían utilizar sin necesidad de ser expertos en el manejo, bastando un nivel medio de conocimientos para que puedan ser utilizados de forma efectiva.

En contraposición a la interpretación anterior y en lo que respecta al acceso a internet de los profesores, solamente el 3,7\% posee disponibilidad permanentemente en sus domicilios; el 74,8\% tiene internet ocasionalmente en centros de cómputo o wi-fi gratuito, y el 21,4\% no tiene ningún tipo de conexión. Estos datos son preocupantes debido a su notable incidencia en la posibilidad de desarrollar eficientemente el proceso de enseñanza-aprendizaje en contextos virtuales. Este antecedente puede ser la causa por la que los maestros no pueden afianzar sus conocimientos sobre el uso de las TIC en la educación, con lo que se justifica la utilización de WhatsApp, a pesar de tener un nivel medio de conocimientos en cuanto al manejo de esas tecnologías.

En lo referente al manejo de entornos virtuales por parte de los estudiantes, según los docentes, solamente $16,7 \%$ tienen un nivel alto, mientras que el $26,8 \%$ un nivel medio y el 56,8\% un nivel bajo. Estos datos se relacionan directamente con el manejo de entornos virtuales de los padres de familia, que presentan un 20,8\% en el nivel alto, $20 \%$ en el medio y $59,2 \%$ en el nivel bajo.

Sobre el proceso de enseñanza-aprendizaje se destaca que el 90\% envía tareas mediante la herramienta que utiliza, en este caso WhatsApp; el 8,3\% realiza clases virtuales y 1,7\% envía tareas impresas. Para evaluar los aprendizajes 87,6\% lo hace mediante actividades enviadas a casa y solamente el 12,4\% desarrolla cuestionarios y/o actividades en línea o plataforma.

En concordancia con los hallazgos obtenidos se evidencia que el 91,8\% de los docentes prefieren impartir clases de forma presencial y/o semipresencial, y solamente el $8,2 \%$ manifiesta su deseo de continuar en la modalidad virtual, lo cual pudiera deberse a la falta de acceso a internet. Estas preferencias perjudican la consolidación de habilidades para aplicar las TIC en entornos educativos virtuales, y obstaculiza la ejecución eficiente del proceso de enseñanza-aprendizaje en el contexto en donde se desarrolla el profesor. 


\subsection{Inteligencia emocional}

En lo que respecta al ámbito de la Inteligencia Emocional se midió el nivel en tres aspectos: 'atención', 'claridad' y 'reparación de emociones'. Según los resultados obtenidos se desprende que los profesores con mayor nivel emocional en cuanto a la 'atención', son los que menos habilidades tienen en el manejo de las TIC. En lo concerniente a 'claridad' la inteligencia emocional se encuentra potenciada en los que mejor manejan las TIC. Finalmente, los que más habilidades tienen en el manejo de las TIC también demuestran mayor nivel de inteligencia emocional en el aspecto 'reparación'. Esto se puede evidenciar en la Tabla 3, en la que se observa que la mayoría de los profesores poseen el nivel medio en cuanto al manejo de las TIC.

\begin{tabular}{|c|c|c|c|c|c|c|c|}
\hline & & \multirow[b]{2}{*}{$\mathbf{N}$} & \multirow[b]{2}{*}{ Media } & \multirow[b]{2}{*}{$\begin{array}{l}\text { Desviación } \\
\text { estándar }\end{array}$} & \multirow[b]{2}{*}{$\begin{array}{l}\text { Error } \\
\text { estándar }\end{array}$} & \multicolumn{2}{|c|}{$\begin{array}{l}\text { 95\% del intervalo } \\
\text { de confianza para la } \\
\text { media }\end{array}$} \\
\hline & & & & & & $\begin{array}{l}\text { Límite } \\
\text { inferior }\end{array}$ & $\begin{array}{l}\text { Límite } \\
\text { superior }\end{array}$ \\
\hline \multirow[t]{3}{*}{ Atención } & Bajo & 31 & 25,87 & 6,682 & 1,200 & 23,42 & 28,32 \\
\hline & Medio & 361 & 24,54 & 6,794 & ,358 & 23,83 & 25,24 \\
\hline & Alto & 93 & 25,24 & 7,581 &, 786 & 23,68 & 26,80 \\
\hline \multirow[t]{3}{*}{ Claridad } & Bajo & 31 & 25,61 & 6,535 & 1,174 & 23,22 & 28,01 \\
\hline & Medio & 361 & 28,27 & 6,448 & 339 & 27,60 & 28,93 \\
\hline & Alto & 93 & 31,02 & 7,460 & ,774 & 29,49 & 32,56 \\
\hline \multirow[t]{3}{*}{ Reparación } & Bajo & 31 & 29,13 & 5,265 & ,946 & $27,2 O$ & 31,06 \\
\hline & Medio & 361 & 30,90 & 5,524 & ,291 & 30,33 & 31,47 \\
\hline & Alto & 93 & 32,14 & 5,974 & ,619 & 30,91 & 33,37 \\
\hline
\end{tabular}

Tabla 3 - Medias de atención, claridad y reparación

En cuanto a la Inteligencia Emocional total, los que mejor manejan las TIC poseen más fortalezas en inteligencia emocional $(\overline{\mathrm{X}}=88,40)$; sin embargo, la mayoría de los docentes se encuentra en el nivel medio de habilidades en el uso de las TIC (361), tal como se observa en la Tabla 4.

\begin{tabular}{|c|c|c|c|c|c|c|c|c|}
\hline & \multirow[b]{2}{*}{$\mathbf{N}$} & \multirow[b]{2}{*}{ Media } & \multirow[b]{2}{*}{$\begin{array}{l}\text { Desviación } \\
\text { estándar }\end{array}$} & \multirow[b]{2}{*}{$\begin{array}{l}\text { Error } \\
\text { estándar }\end{array}$} & \multicolumn{2}{|c|}{$\begin{array}{l}\text { 95\% del intervalo } \\
\text { de confianza para la } \\
\text { media }\end{array}$} & \multirow[b]{2}{*}{ Mínimo } & \multirow[b]{2}{*}{ Máximo } \\
\hline & & & & & $\begin{array}{l}\text { Límite } \\
\text { inferior }\end{array}$ & $\begin{array}{l}\text { Límite } \\
\text { superior }\end{array}$ & & \\
\hline Bajo & 31 & 80,61 & 15,760 & 2,831 & 74,83 & 86,39 & $5^{2}$ & 105 \\
\hline Medio & 361 & 83,71 & 14,861 &, 782 & 82,17 & 85,24 & 46 & 116 \\
\hline Alto & 93 & 88,40 & 17,714 & 1,837 & 84,75 & 92,05 & 33 & $12 O$ \\
\hline Total & 485 & 84,41 & 15,610 & ,709 & 83,02 & 85,80 & 33 & $12 O$ \\
\hline
\end{tabular}

Tabla 4 - Media Inteligencia emocional total 
Al comparar los niveles de inteligencia emocional por sexo, se observa que los hombres tienen un mayor puntaje $(\overline{\mathrm{X}}=84,70)$, que el presentado por las mujeres $(\overline{\mathrm{X}}=84,21)$; es decir, el sexo masculino posee una Inteligencia Emocional más alta, aunque sin diferencias significativas, tal como se evidencia en la Tabla 5.

\begin{tabular}{llllll}
\hline & Sexo & N & Media & $\begin{array}{l}\text { Desviación } \\
\text { estándar }\end{array}$ & $\begin{array}{l}\text { Media de error } \\
\text { estándar }\end{array}$ \\
\hline \multirow{2}{*}{$\begin{array}{l}\text { Inteligencia emocional } \\
\text { total }\end{array}$} & Masculino & 199 & $\mathbf{8 4 , 7 0}$ & 16,786 & 1,190 \\
\cline { 2 - 6 } & Femenino & 286 & $\mathbf{8 4 , 2 1}$ & 14,763 &, 873 \\
\hline
\end{tabular}

Tabla 5 - Medias de Inteligencia Emocional, según el sexo

\subsection{Correlación entre habilidades para el manejo de las TIC y la inteligencia emocional}

\begin{tabular}{|c|c|c|c|c|}
\hline & & & $\begin{array}{l}\text { Manejo de TIC } \\
\text { en entornos } \\
\text { virtuales }\end{array}$ & $\begin{array}{l}\text { Inteligencia } \\
\text { emocional total }\end{array}$ \\
\hline \multirow[t]{6}{*}{$\begin{array}{l}\text { Rho de } \\
\text { Spearman }\end{array}$} & \multirow[t]{3}{*}{$\begin{array}{l}\text { Manejo de TIC en } \\
\text { entornos virtuales }\end{array}$} & $\begin{array}{l}\text { Coeficiente de } \\
\text { correlación }\end{array}$ & 1,000 &, $\mathbf{1 3 4} 4^{* *}$ \\
\hline & & Sig. (bilateral) & . &, $\mathbf{0 0 3}$ \\
\hline & & $N$ & 485 & 485 \\
\hline & \multirow[t]{3}{*}{$\begin{array}{l}\text { Inteligencia } \\
\text { emocional total }\end{array}$} & $\begin{array}{l}\text { Coeficiente de } \\
\text { correlación }\end{array}$ &, $134^{* *}$ & 1,000 \\
\hline & & Sig. (bilateral) & ,oo3 & . \\
\hline & & $N$ & 485 & 485 \\
\hline
\end{tabular}

Tabla 6 - Correlación TIC-Inteligencia Emocional

Para determinar la asociación entre las habilidades para el uso de las TIC en educación virtual y la Inteligencia Emocional, se utilizó el coeficiente Rho de Spearman. Tal como se observa en la Tabla 6, los resultados obtenidos permiten afirmar que sí existe correlación entre las variables planteadas, pues se observa un nivel de significación de o,o03; sin embargo, la asociación es muy baja y positiva $(, 134)$, lo que conlleva a deducir que mientras más altas sean las habilidades para el manejo de las TIC mayor será también el nivel de Inteligencia Emocional.

\section{Conclusiones}

En este estudio se identificó el nivel de conocimiento y uso de las Tecnologías de Información y Comunicación (TIC) de los docentes en contextos virtuales de educación. $\mathrm{Al}$ respecto, la mayoría de los educadores que participaron en el estudio posee un nivel medio de conocimiento de las TIC, pero existen limitaciones que dificultan su labor profesoral; destacando entre ellas: (a) la falta de capacitación y cualificación docente, 
(b) la limitada o incluso nula disponibilidad de un alto número de maestros para acceder a internet, y (c) las pocas habilidades de los padres de familia y los estudiantes para el manejo de los entornos virtuales educativos. Por otra parte, cabe señalar que ante la precariedad de la infraestructura virtual para fomentar y alcanzar eficientemente los objetivos del proceso de enseñanza-aprendizaje, los maestros intentan responder a las necesidades educativas mediante el uso de aplicaciones alternativas, como WhatsApp, la cual se ha transformado en fuente primaria de comunicación durante el desarrollo del proceso educativo.

En lo que respecta al nivel de Inteligencia Emocional de los profesores, se destaca que existe una tendencia hacia los niveles medio y bajo en cuanto al manejo de las emociones estudiadas, lo cual se relaciona directamente con el manejo de las TIC en entornos virtuales. Estas circunstancias inciden directamente sobre el desarrollo del proceso de enseñanza-aprendizaje en el contexto de la emergencia sanitaria.

Finalmente, se demostró que existe correlación positiva, aunque baja, entre las habilidades para el manejo de las TIC y la Inteligencia Emocional de los profesores, lo cual da a entender que en la medida que el docente posea más conocimientos y habilidades para el manejo de estas tecnologías en educación virtual, también será mayor su fortaleza emocional, y viceversa.

\section{Referencias}

Alemany, D., \& Martínez, A. (2017). Ámbitos de actuación en entornos virtuales de aprendizaje de idiomas: Propuesta de una lista de verificación basada en criterios de calidad. En Innovación docente y uso de las TIC en educación (s/n), 1-11. http://www.enriquesanchezrivas.es/congresotic/archivos/CampusVirtuales/ Alemany_Otros.pdf

Balladares-Burgos, J. (2018). Diseño pedagógico de la educación digital para la formación del profesorado. Revista Latinoamericana de Tecnología Educativa, 17(1), 60-41. http://dx.medra.org/10.17398/1695-288X.17.1.41

Bar-On, R., \& Parker, J. (2000). The emotional quotient inventory: Youth version. Multi-Health Systems.

Bolívar, C. R., \& Dávila, A. A. (2016). Propuesta de buenas prácticas de educación virtual en el contexto universitario. Revista de Educación a Distancia (RED), 49(12), 1-21. http://dx.doi.org/10.6018/red/49/12

Cabanillas, J., Veríssimo, S., \& Luengo, R. (2020). Contraste en la percepción sobre el uso de una plataforma virtual para la mejora de la enseñanza y aprendizaje de las matemáticas. RISTI - Revista lbérica de Sistemas e Tecnologias de Informação, (38), 33-47. https://doi.org/10.17013/risti.38.33-47

Espinoza-Venegas, M., Sanhueza-Alvarado, O., Ramírez-Elizondo, N., \& Sáez-Carrillo, K. (2015). A validation of the construct and reliability of an emotional intelligence scale applied to nursing students. Revista Latino-Americana de Enfermagem, 23(1), 139-147. https://doi.org/10.1590/0104-1169.3498.2535 
García, C. H. (2016). Inteligencia Emocional y Bienestar. En Inteligencia emocional y bienestar II (32-44). Ediciones Universidad de San Jorge. https://dialnet.unirioja. es/servlet/articulo?codigo $=5568420$

Goleman, D. (2007). La Inteligencia Emocional. ZETA.

Goleman, D. (2010). La práctica de la inteligencia emocional. Editorial Kairós.

Goleman, D. (2018). Inteligencia emocional en la empresa (Imprescindibles). Penguin Random House Grupo Editorial.

Gómez, D., \& Trigueros, I. M. (2017). Las WebQuests y los MOOCs en la enseñanza de las Ciencias Sociales y la formación del profesorado de Educación Primaria. Revista Electrónica Interuniversitaria de Formación del Profesorado, 20(2), 205-220. https://doi.org/10.6018/reifop/20.2.258551

Granados, J., Vargas, C., \& López, R. (2017). Estrategia de formación continua del docente universitario en la didáctica de los entornos virtuales de aprendizaje (EVA). Revista Conrado, 13(1), 78-86.

Hallo, A., Rojas, A., \& Hallo, C. (2020). Perspective from Ecuador, the Second Country with More Confirmed Cases of Coronavirus Disease 2019 in South America: A Review. Cureus, 12(3), 2-6. https://doi.org/10.7759/cureus.7452

Hernández, R., Fernández, C., \& Baptista, M. (2014). Metodología de la Investigación (Sexta edición). McGraw Hill Education.

Inca, G. P., \& Inca, A. C. (2020). Evolución de la enfermedad por coronavirus (COVID-19) en Ecuador. La Ciencia al Servicio de la Salud, 11(1), 5-15.

López, C. C., Bulla, N. A., Gómez, P., \& Rodríguez, D. (2019). Formación Virtual: Enseñanza y Aprendizaje de la Probabilidad. En Tendencia Reciente en la Educación Probabilística y Estadística (s/n), 35-51. Benemérita Universidad Autónoma de Puebla. http://funes.uniandes.edu.co/14272/

Manrique-Losada, B., Gómez-Álvarez, M., \& González-Palacio, L. (2020). Estrategia de transformación para la formación en informática: Hacia el desarrollo de competencias en educación básica y media para la Industria 4.0 en Medellín Colombia. RISTI - Revista lbérica de Sistemas e Tecnologias de Informação, (39), 1-17. https://doi.org/10.17013/risti.39.1-17

Marciniak, R., \& Gairín, J. (2018). Dimensiones de evaluación de calidad de educación virtual: Revisión de modelos referentes. RIED. Revista Iberoamericana de Educación a Distancia, 21(1), 217-238. https://doi.org/10.5944/ried.21.1.16182

Merchán, I., \& González, J. (2012). Análisis de la eficacia de un Programa de Inteligencia Emocional con profesores de Badajoz y Castelo Branco (Portugal). Campo abierto: Revista de educación, 31(1), 51-68.

Mesa, G. H., Blanco, M. R., \& Addine, R. (2018). La virtualización del contenido nomenclatura química en la educación superior pedagógica. Revista de Investigación en Tecnologías de la Información: RITI, 6(12), 84-93. 
Mogollón, I., Medina, C., \& Correa, K. (2017). Desarrollo de experiencias de aprendizaje virtual accesible: Atención a las necesidades de personas con discapacidad visual. Edutec. Revista Electrónica de Tecnología Educativa (62), 34-47. https://doi.org/10.21556/edutec.2017.62.1023

Organización Mundial de la Salud. (2020). Organización Mundial de la Salud: Según una encuesta mundial de la OMS, el 90\% de los países han sufrido interrupciones de sus servicios de salud esenciales desde el inicio de la pandemia de COVID-19. https://www.who.int/es

Renés, P. (2018). Planteamiento de los estilos de enseñanza desde un enfoque cognitivoconstructivista-Dialnet. Tendencias pedagógicas (31), 47-68.

Rodríguez, R. (2016). Exigencias éticas de la calidad de la educación virtual. En El aseguramiento de la calidad de la educación virtual (pp. 55-68). ULADECH.

Rodriguez-Morales, A. J., Sánchez-Duque, J. A., Botero, S., Pérez-Díaz, C. E., VillamilGómez, W. E., Méndez, C. A., Verbanaz, S., Cimerman, S., Rodriguez-Enciso, H. D., Escalera-Antezana, J. P., Balbin-Ramon, G. J., Arteaga-Livias, K., CvetkovicVega, A., Orduna, T., Savio-Larrea, E., \& Paniz-Mondolfi, A. (2020). Preparación y control de la enfermedad por coronavirus 2019 (COVID-19) en América Latina. Acta Médica Peruana, 37(1), 3-7. https://doi.org/10.35663/amp.2020.371.909

Salovey, P., Mayer, J., Goldman, S., Turvey, C., \& Palfai, T. (1995). Emotional attention, clarity, and repair: Exploring emotional intelligence using the Trait Meta-Mood Scale. En Emotio, Disclosure, y Health (J. Pennebaker, pp. 125-151). American Psychological Association.

Suasnabas, L., Quinto, E. D., \& Alcázar, J. A. (2018). Impacto de las aulas virtuales en el sistema de educación superior de Ecuador. RECIAMUC, 2(1), 945-959.

Ugarriza, N., \& Pajares-Del-Águila, L. (2005). La evaluación de la inteligencia emocional a través del inventario de BarOn ICE: NA, en una muestra de niños y adolescentes. Persona (8), 11-58. https://doi.org/10.26439/persona2005.noo8.893

Zambrano-Vacacela, L., \& Yautibug-Chimbolema, M. (2020). Modelo AIM para la enseñanza-aprendizaje con estudiantes de modalidad semipresencial-distancia en la Universidad Nacional de Educación. Killkana sociales: Revista de Investigación Científica, 4(1), 13-20. 